Research Article

\title{
Estimation Method of Inundation Extent Based on Mathematical Morphology
}

\author{
Jianxun Li $\mathbb{D},{ }^{1}$ Kin Keung Lai $\left(\mathbb{D},{ }^{2}\right.$ and Paul Safonov $\mathbb{D i}^{3}$ \\ ${ }^{1}$ School of Economics and Management, Xi'an University of Technology, Xi'an 710048, China \\ ${ }^{2}$ College of Economics, Shenzhen University, Shenzhen 518060, China \\ ${ }^{3}$ Herberger Business School, St. Cloud State University, St. Cloud, MN 56301, USA \\ Correspondence should be addressed to Kin Keung Lai; mskklai@outlook.com
}

Received 16 June 2019; Revised 30 August 2019; Accepted 23 September 2019; Published 16 October 2019

Academic Editor: Ioannis Kostavelis

Copyright (C) 2019 Jianxun Li et al. This is an open access article distributed under the Creative Commons Attribution License, which permits unrestricted use, distribution, and reproduction in any medium, provided the original work is properly cited.

Estimation of inundation extent is critical for the flood control system. It helps decision-maker determine the scope of damage to save residents' lives and property. In this paper, we analyzed the drawbacks of two kinds of methods of inundation estimation, hydrodynamic method and space-filling method, and divided inundation extent into water-body extent and water-invasion extent for the purpose of enhancing estimation accuracy. Based on Mathematical Morphology, an inundation estimation method suitable for flood mapping around natural and artificial lakes was put forward by integrating remote sensing (RS) data and digital elevation model (DEM) data. This method consists of three aspects, including the subdivision of water-body extent, the deduction of water-invasion extent which is viewed as the expansion of water-body extent, and the smoothing the boundaries of waterinvasion extent. With subdividing DEM data horizontally into cross sections to build equiangular subsets, the expansion operation was implemented by structuring elements which were established based on set operations and scalar flexibilities. Thus, water-invasion extent was easily described by the structuring elements under given water volume, so that inundation extent was conveniently estimated by spatial data. The experiment results show that the method has the advantages of high accuracy and expressiveness and low time consumption. It had been used to analyze the inundation caused by the water conservancy project named Hanjiang-to-Weihe River Water Transfer Project in China. The method served as decision support for building an efficient emergency response mechanism and helped implement flood disaster forecast and simulation.

\section{Introduction}

Inundation is result from flood and tsunami and even from the construction of hydraulic projects that cuts off river to build a dam. Viewed as a distinct model of catastrophe, inundation usually poses threats to mankind's existence and development. The United Nations Office for Disaster Risk Reduction (UNDRR) reports that inundation accounts for over $30 \%$ of all natural disaster losses. Inundation appears to be a particular serious problem in crowded China due to its long time span, wide range, unexpected frequency, and tremendous devastation. Statistically, China has long been harassed by inundations and is confronted with catastrophic flood every two years. Approximately $28 \%$ of arable land and $46 \%$ of the population nationwide are subject to the detrimental effect of inundations, thus hampering the sustainable development of economy and society. In the process of recovering from these damages, inundation estimation plays a significant role in analyzing the affected extent and the loss amount. It forecasts inundation extent, marks the flood-stricken region, and gives emergency response instructions.

For the convenience of analysis and estimation, we divided the inundation extent into water-body extent and water-invasion extent. Water-body extent, inundating lowlying region below water surface elevation, is related to the regional terrain and water volume. Water-invasion extent, namely, the region adjacent to water-body extent, is generally determined by water surface elevation, terrain, and geological conditions. Considering the inaccuracy of most water-body extent estimations and the difficulty in waterinvasion extent measurement, we regarded water-invasion 
extent as the expansion of water-body extent, due to the fact that water-invasion extent produced by capillary rise is the saturated portion of the soil surrounding water-body extent. Then, a more accurate estimation method combining spacefilling method and RS data was proposed for inundation estimation by introducing the Mathematical Morphology. The method is mainly suitable for flood mapping around natural and artificial lakes. We has implemented the method on Digital Earth platform and formulated a prototype system which could analyze the affected extent in real time.

The remainder of the paper is organized as follows. Section 2 briefly reviews related work on inundation estimation. Section 3 presents an estimation method for inundation depth and inundation extent based on Mathematical Morphology. In Section 4, an experiment environment with the Digital Earth platform is built to verify the effectiveness of the method. Section 5 concludes the paper and provides remarks.

\section{Literature Review}

The estimation method of inundation extent is currently divided into two categories. One is hydrodynamic method which simulates the depth distribution of water mobility, resolves continuity equations and motion equations, and then estimates inundation extent by the evolution of unsteady flow. This method requires calculating partial differential equations with multiple parameters. The other one is space-filling method which takes DEM as basic data. With the help of GIS or RS, it employs a differential method to decompose the research area into lots of differential units in the light of certain elevation. The water of these units is dynamically accumulated until the discrepancy between cumulative result and water volume is less than the specific threshold representing the estimation accuracy.

2.1. Hydrodynamic Method. Hydrodynamic method integrates hydrology and hydrodynamics to build a model of flood routing by using equations of continuity and motions. It takes into account the characteristics of water transmission, river bed evaluation, roughness coefficient, and turbulent viscosity coefficient. Therefore, the unsteady flow and its variation occurred at different inundating time is easily simulated. Based on hydrodynamics, Horritt et al. [1] constructed an assessment method involving spatial scale. It provided prototype solutions for inundation estimation. Combining the flood extent information derived from RS imagery and hydrodynamic simulations, Nguyen et al. [2] proposed a model for estimating inundation depth and spatially distributed water level. The model provided more effective assessment for local-scale pluvial flood. Due to its strong feasibility, hydrodynamic model has fully been applied to the assessment of inundation extents, e.g., Bates et al. [3] and Podhoranyi et al. [4]. In recent years, researchers mainly studied on the estimation accuracy, computation speed, and simulation environment of inundation. As to the estimation accuracy, Ilorme [5] discussed the influence of the inaccuracy of hydrological data and the insufficiency of hydrometrical stations based on the investigation into the homogeneity in inundation extent. For quickly estimating inundation at hyperresolutions, Follum et al. [6] put forward a routing application for parallel computation of discharge, which can be applied quickly over very large spatial extents. In order to build a simulation environment, Murphy et al. [7] introduced a hydraulic and hydrologic modeling software, Infoworks ICM (Integrated Catchment Modelling), into flood inundation. The software used unstructured triangular mesh and advanced numerical scheme, which improved the simulation speed and calculation accuracy. In addition, there have been several studies aiming at the subproblem of inundation estimation by hydrodynamic method. For example, Gobeyn et al. [8] took LISFLOOD-FP model as an explicit forward difference scheme for computing the shallow water wave over the floodplain. Patel et al. [9] carried out a simulation work under the 1D/2D couple hydrodynamic modeling in low-lying areas. Faghih et al. [10] applied nonparametric bootstrap sampling to simulate inundation with different flow rates, which provided a reference for the uncertainty analysis of inundation.

Above all, under the conservation laws of specific water volume, hydrodynamic method fully demonstrates hydrological characteristics and serves as an accurate estimation model to estimate inundation extent. Relying on partial differential equations, the method obtains a relatively precise estimation of inundation extent and provides a vivid expression of water-body extent, furthermore clearly describing the dynamic features of inundation. However, the model usually contains plenty of parameters and complex equations proved hard to solve [11]. In particular, most existing studies fail to take into consideration the boundary terrain, soil conditions, vegetation, and the water absorption by the earth in water-invasion extent estimation.

2.2. Space-Filling Method. Space-filling method approximately simulates the ultimate equilibrium of inundation by viewing the water surface as a plane. Based on the overland runoff model, this method estimates inundation extent as well as inundation depth [12], such as the automatic integrated estimation approach by Noman et al. [13]. Since space-filling method is implemented by simple mathematical calculations with large errors, the research mainly focuses on improving the estimation accuracy. Dewan et al. [14] earlier used airborne laser altimetry for analyzing inundation extent. It leads researchers to introduce geospatial data into space-filling method. Geospatial data, such as GIS, RS, and DEM, can offer more possibilities for increasing the accuracy of inundation estimation. One of the representative methods was proposed by Mason et al. [15] who adopted RS data to rectify the traditional estimation model. With the combination of flooding data and RS data, Kamontum [16] applied Landsat and IRS images to inundation extent analysis, further improving the estimation of inundation boundary. Recently, scholars conducted in-depth research on the estimation model of DEM data or cellular automata approach [17, 18]. Saksena and Merwade [19] considered that vertical accuracy is related to the spatial resolution 
errors of DEM properties. Teng et al. [20] developed and implemented a floodplain inundation model that uses highresolution DEM to derive floodplain storages and connectivity between them at different river stages. Moreover, with the support of interpolation techniques, Sopelana et al. [21] proposed a continuous simulation method for the estimation of extreme inundation in coastal river reaches. It enhanced the effectiveness and practicability of space-filling method in inundation estimation.

To sum up, space-filling method is not as accurate as hydrodynamic method but convenience to calculation. Depending on digital elevation, the method uses total water volume and elevation data to conduct alignment analysis. The accuracy of space-filling method could be considerably enhanced when the digital elevation has high accuracy and the connected regions become easy to identify. However, due to the lack of the ability to make full use of spatial data, the method fails to manifest the terrain factors and calculate water-invasion extent. The inundation extent estimated by the method is usually smaller than the actual extent since it does not distinguish between inundation extent and waterinvasion extent.

Except the two categories of hydrodynamic method and space-filling method, there are some other methods mentioned by researchers, such as empirical method, simplified method [22], and Bayesian statistical method [23]. However, looking at the results of existing researches, they mostly ignore the estimation of waterinvasion extent and only regard water-body extent as inundation extent because it is difficult to describe waterinvasion extent which is related to the boundary of water, terrains, and landforms. Thus, incorporating the Mathematical Morphology [24], we proposed an inundation estimation method by combined space-filling method and RS data. The method divided inundation extent into water-body extent and water-invasion extent with high timeliness and accuracy. It is worth mentioning that Vernieuwe et al. [25] have put forward a Mathematical Morphology approach for a qualitative exploration of drought events in space and time. This study demonstrates that the expansion operation and erosion operation of Mathematical Morphology can be used to analyze water-related problems. More importantly, Li et al. [26] have presented an inundation estimation method using the Mathematical Morphology algorithm, but they adopted simple structuring elements and lack the consideration of water-invasion issues. Therefore, the accuracy of inundation extent analysis still has a chance to be improved.

\section{Inundation Estimation Based on Mathematical Morphology}

As a theory for obtaining intrinsic characteristics by structuring elements, Mathematical Morphology is widely used in image processing, artificial intelligence, shape analysis, and topology research. It can extract the shape of the object and explore the morphologic changes under a variety of conditions, thus providing a basic evolutionary method tool between different structures. In this paper, we divide the inundation extent into water-body extent and water-invasion extent. Water-invasion extent, absorbing certain portion of water by soil and vegetation, is the expansion of water-body extent. Due to the complexity of water-invasion process, we introduced Mathematical Morphology so as to obtain more accurate waterinvasion extent and relatively high calculate speed. Correspondingly, we estimated water-body extent by the modified space-filling method and horizontally subdivided it for conveniently implementing expansion operation.

\subsection{Introduction of Mathematical Morphology.} Mathematical Morphology derives from set theory. It gives up traditional ways of mathematical analysis and modeling and carries out data interpretation from the perspective of aggregation. Moreover, it probes into geometry data by using structuring element which supplies various data, such as direction, size, and chrominance. Due to its capacity of describing geometrical morphology, we used the geometrical description to analyze the topographic elements of inundation extent. Here, inundation extent was regarded as a binary image set, and water-invasion extent was accordingly considered as the expansion of water-body extent based on local landforms. Geographically, water-invasion extent is formed by the capillary rise of the soil surrounding water-body extent. The capillary rises in different landforms are, respectively, expressed as different evolutions of geometrical structures. Therefore, we described the expansion caused by capillary rise in a way of original image processing by structuring elements, so that the relationship between water-body extent and water-invasion extent was established by Mathematical Morphology, and inundation extent was precisely estimated based on the operations of structuring elements, such as horizontal panning, crossing, and merging.

Suppose $A$ denotes the binary digital image set of waterbody extent and $A^{C}$ the complementary set of $A$. Then, the translation action $A[X]$ according to vector $X$ and the reflection action $-A$ according to coordinate origin are shown as follows:

$$
\begin{aligned}
A[X] & =\{a+x \mid a \in A\}, \\
-A & =\{-a \mid a \in A\} .
\end{aligned}
$$

Based on these definitions of Mathematical Morphology, the erosion operation of contraction process in water-invasion extent is described by

$$
A \Theta B=\{x \mid B+x \subset A\}=\bigcap\{A-b \mid b \in B\}=\bigcap_{b \in B} A[-b] .
$$

Here, $B$ is a structuring element or a contraction template of water-invasion extent, and the points in $A \Theta B$ indicate the template's original location gained by panning $B$ in $A$. In other words, $A \Theta B$ means the result of intersection operation through panning $A$ by $-b\{b \in B\}$. As an inverse operation of erosion operation, expansion operation is defined as 


$$
\begin{aligned}
A \oplus B & =\{x \mid(-B+x) \cap A \neq \Phi\}=\bigcup\{A+b \mid b \in B\} \\
& =\bigcup\{a+b \mid a \in A, b \in B\}=\bigcup_{b \in B} A[b] .
\end{aligned}
$$

The expansion operation expands the specific areas of the binary image by adding pixels to the perceived boundaries and satisfies the following equation:

$$
\begin{aligned}
& (A \oplus B)^{C}=A^{C} \Theta(-B), \\
& (A \Theta B)^{C}=A^{C} \oplus(-B),
\end{aligned}
$$

where $A \oplus B$ is obtained through panning $A$ by $-b\{b \in B\}$, which facilitates the expansion of $A$ by $B$.

Thus, water-invasion extent corresponding to waterbody extent $A$ can be estimated by expansion operation, if appropriate structuring elements are chosen. Besides erosion operation and expansion operation, there is another important action named opening operation in Mathematical Morphology. The opening operation is a quadratic formulation combining erosion operation and expansion operation defined as

$$
A \circ B=(A \Theta B) \oplus B=\bigcup\{B+X \mid B+X \subset A\} .
$$

It is acquired by structuring elements that fill in the interior of water-invasion extent image. The opening operation as a geometric process in water-invasion extent is used to delete useless structuring elements, smooth extent outline, cut off narrow connection, and eliminate tiny or projecting part. That is to say, opening operation can connect neighboring fields or smooth borders and improve the expressiveness of inundation extent.

In addition, the erosion operation and expansion operation in Mathematical Morphology not only have the monotonicity characteristics of $\left(A^{\prime} \subseteq A \Longrightarrow A^{\prime} \Theta B \subseteq A \Theta B, A^{\prime} \oplus B \subseteq A \oplus B\right)$ and the expansibility characteristics of $(A \Theta B \subseteq A \subseteq A \oplus B)$, but have translation invariance:

$$
\begin{aligned}
A[X] \Theta B & =(A \Theta B)[X], \\
A[X] \oplus B & =(A \oplus B)[X], \\
A \Theta B[X] & =(A \Theta B)[-X], \\
A \oplus B[X] & =(A \oplus B)[X] .
\end{aligned}
$$

It indicates that water-body extent or templates used for conducting erosion operation and expansion operation can change the position instead of the shape of waterinvasion extent. Thus, we can use a template expanding water-body extent to water-invasion extent, as long as different locations share the same terrain properties. More importantly, the expansion operation conforms to commutative law $A \oplus B=B \oplus A$ and associative law $A \oplus$ $(B \oplus C)=(A \oplus B) \oplus C$, and the large-scale structuring elements $D$ can be decomposed to $D_{1} \oplus D_{2} \oplus, \ldots, \oplus D_{n}$, thus making it possible for small-scale structuring elements to replace large-scale ones. Moreover, the expansion operation also has scale flexibility: when the scalar product is $t A=\{t a \mid a \in A\}$, for any real number $t$, there are five set operations as

$$
\begin{aligned}
t(A \oplus B) & =t A \oplus t B, \\
t(A \oplus B) & =t A \oplus t B, \\
(A \cap B) \oplus C & =(A \oplus C) \cap(B \oplus C), \\
(A \cup B) \oplus C & =(A \oplus C) \cup(B \oplus C), \\
A \oplus(B \cup C) & =(A \oplus B) \cup(A \oplus C) .
\end{aligned}
$$

This indicates further that the expansion operation of water-body extent can be divided into different subset operations conducted by structuring elements. The function of subset operations is to carry out morphological processing independently and achieve eventual results through set operations.

3.2. Subdivision of Water-Body Extent. The estimation of water-body extent is only subject to the water volume in inundation extent and the landform of the underlying surface. With DEM data, space-filling method is usually used to estimate the region (water-body extent) obviously covered with water. It vertically subdivides the research area into cubic columns with different depths. However, water-invasion extent as the expansion of water-body extent is a horizontal and outward operation in size and range, so the existing methods hardly describe the relevance of waterinvasion extent to water-body extent. Due to the invariance of water-body subdivided vertically or horizontally, we use a cross-section set $X$ to horizontally subdivide water-body extent. As shown in Figure 1, if $\Delta h$ and $H$ represent the elevation interval and the depth of water-body extent, the cross-section set can be denoted as $X=\left\{X^{0}, X^{\Delta h}\right.$, $\left.X^{2 \Delta h}, \ldots, X^{\lambda \Delta h}\right\}$, where water-body extent is subdivided by $\lambda=H / \Delta h$ times. If $\lambda$ is big enough, the water volume $V_{m}$ in water-body extent in fact is the accumulation of those cross sections. Thus, we have

$$
V_{m}=\int_{0}^{H} S\left(X^{\eta}\right) d \eta \approx \sum_{i=0}^{\lfloor H / \Delta h\rfloor} S\left(X^{i \Delta h}\right) \Delta h,
$$

where $X^{\eta}$ denotes the specific cross section of water-body extent at depth $\eta$. The area of $X^{\eta}$ is represented as $S\left(X^{\eta}\right)$, where $S(\cdot)$ is an area operator. Since the cross-section set $X$ is a series of intersections between the horizontal planes with depth $\{0, \Delta h, 2 \Delta h, \ldots, \lambda \Delta h\}$ and DEM data, we can easily obtain water-body extent as long as we find depth $H$ that satisfies equation (8). In other words, water-body extent is equal to the area of intersection between DEM data and horizontal planes at depth $H$. Here, dichotomy is a simple way to get the suitable depth $H$ when the water volume $V_{m}$ is given, which is as same as traditional space-filling method.

Usually, water-body extent is over several square kilometers. Its border is too large to directly deduce for waterinvasion extent by Mathematical Morphology. So we divided water-body extent into different subset operations that were easily implemented by structuring elements. Here, structuring elements are the template components used to expand water-invasion extent from water-body extent. The shape of structuring elements is related to landform factors such as geological condition, soil, vegetation, and boundary of 


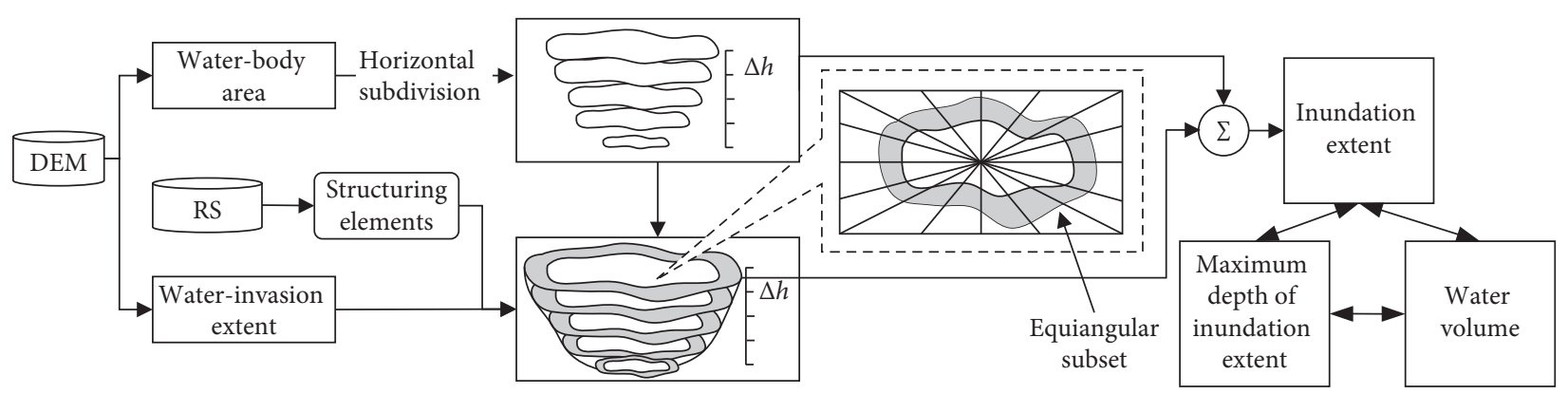

FIGURE 1: Subdivision of water-body extent.

water-body extent. Obviously, it is difficult to construct structuring elements directly on account of the influence of these factors. A relatively comprehensive method is to collect the data of landforms and test the water absorption ability through experiments. This method helps to establish structuring elements with high accuracy, but lots of experimentation with various types of boundaries and landforms lead to a great amount of prework, which significantly increases time consumption. For improving the response speed of Flood Control Decision Support System, we assume that two neighboring landforms with sufficiently small boundaries shared homogeneous properties since the geological conditions in the sufficiently small region are similar and water bodies usually do not cross different geological belts. Thus, the typical structuring elements under different elevations can be obtained according to the boundaries and landforms of water-body extent. Suppose the landform factors denote $\varphi_{i}=\left\{\varphi_{i}^{1}, \varphi_{i}^{2}, \ldots, \varphi_{i}^{\mu}\right\}$ gained from RS data, then $\varphi_{i}$ can be normalized to $\psi_{i}=\left\{\psi_{i}^{1}, \psi_{i}^{2}, \ldots, \psi_{i}^{\mu}\right\}$ and the aggregation of ${ }^{j}$ all landform factors by OWG operator is $f_{i}=\prod_{j=1}^{\mu}\left[\phi_{i}^{j}\right]^{\omega^{j}}$, where $\psi_{i}^{j}$ indicates $\varphi_{i}^{j} / \sum_{i=0}^{n} \varphi_{i}^{j}, \phi_{i}^{j}$ denotes the $j$ biggest data of $\psi_{i}$, and $\left\{\omega^{1}, \omega^{2}, \ldots, \omega^{\mu}\right\}$ is the weights satisfied with $\sum_{i} \omega_{i}=1$. According to Mathematical Morphology, the similar set $E_{i}=\left\{E_{i, j}\right\}$ will be the structuring elements related to the expansion operation from waterbody extent to the water-invasion extent, where $E_{i, j}=\operatorname{sim}\left(f_{i}, f_{j}\right)=f_{i} \cdot f_{j} /\left(\left|f_{i}\right|\left|f_{j}\right|\right)$ is the similarities of vectorial angle cosine. This morphology operation by structuring element is different from the 8-direction structuring element method merely used to implement the expansion. It can also be applied to different elevations though it is worth noting that higher pressure can lead to more water invasion and absorption at the boundaries of water-body extent.

3.3. Morphological Estimation of Inundation Extent. Since inundation extent is composed of water-body extent and water-invasion extent, there are three steps needed for the estimation of inundation extent based on morphology: expanding water-body extent into water-invasion extent, estimating the depth of inundation extent based on the given volume of water, and smoothing the boundaries of waterinvasion extent to improve the visualization effect of inundation simulation. (i) Suppose $Y^{\eta}$ denotes the water-invasion extent corresponding to water-body extent $X^{\eta}$ at depth $\eta$. According to the characteristics of morphology operations, $X^{\eta}$ and $Y^{\eta}$ can be subdivided into equiangular subsets $X^{\eta}=X_{1}^{\eta} \cup X_{2}^{\eta}, \ldots, \cup X_{n}^{\eta}$ and $Y^{\eta}=Y_{1}^{\eta} \cup Y_{2}^{\eta}, \ldots, \cup Y_{n}^{\eta}$ in polar coordinate system, where $X_{i}^{\eta}$ and $Y_{i}^{\eta}$ share the equal angles. In Mathematical Morphology, the structuring elements for expansion operation from $X_{i}^{\eta}$ to $Y_{i}^{\eta}$ can be represented by a set $C^{\eta}=C_{1}^{\eta} \cup C_{2}^{\eta}, \ldots, \cup C_{n}^{\eta}$. Let $E_{i}$ defined in Section 3.2 denote the expansion structuring elements under the condition of homogeneous properties, then $C_{i}^{\eta}$ and $C_{i}^{\xi}, \xi \in\{0, \Delta h, \ldots, \lambda \Delta h\}$ is described as $a^{\eta} E_{i}$ and $C_{i}^{\xi}=C_{i}^{\eta} a^{\xi} / a^{\eta}$, where $a^{\eta}=k \int_{0}^{\eta} S\left(X^{\eta}\right) \mathrm{d} \eta$ is the osmotic pressure generated by water body at depth $\eta$ and $k$ is the coefficient denoting the osmotic pressure per unit of water volume. The coefficient $k$ is related to the bulk density, porosity, and infiltration of the soil surrounding the water-body extent. Due to the similarity of geological conditions in a small region, the value of $k$ does not change in a certain area and can be obtained from the soil property dataset. Meanwhile, the water-invasion extent can be deduced according to set operations and scalar flexibilities of expansion operation. In other words, $Y^{\eta}=$ $\cup_{i}\left(X_{i}^{\eta} \oplus C_{i}^{\eta}\right)=a^{\eta} \cup_{i}\left(X_{i}^{\eta} \oplus C_{i}\right)$ is the water-invasion extent corresponding to water-body extent $X^{\eta}$.

(ii) Assume the depth of inundation is $H$ and the coefficient integrating permeability and absorption rates (namely, the water volume per unit area of water-invasion extent) is $b$. Since the depth of waterbody extent, water-invasion extent, and inundation extent are the same, the water-body below depth $H$ can be represented by the aggregation of the water volume of water-body extent $V_{m}=\int_{0}^{H} S\left(X^{\eta}\right) \mathrm{d} \eta$ and that of water-invasion extent:

$$
\begin{aligned}
V_{x} & =b \int_{0}^{H} S\left(Y^{\eta}\right) \mathrm{d} \eta=\frac{b}{a^{\eta}} \int_{0}^{H} a^{\xi} S\left(\bigcup_{i}\left(X_{i}^{\xi} \oplus C_{i}\right)\right) \mathrm{d} \xi \\
& =\frac{b}{\int_{0}^{\eta} S\left(\bigcup_{i} X^{\eta}\right) \mathrm{d} \eta} \int_{0}^{H}\left(\int_{0}^{\xi} S\left(X^{\eta}\right) \mathrm{d} \eta\right) S\left(\bigcup_{i}\left(X_{i}^{\xi} \oplus C_{i}\right)\right) \mathrm{d} \xi .
\end{aligned}
$$


Thus, the water volume of inundation extent is as follows:

$$
\begin{aligned}
V_{H}= & V_{m}+V_{x} \approx \sum_{j}^{\lfloor H / \Delta h\rfloor} S\left(X^{j \Delta h}\right) \Delta h+\frac{b}{\sum_{j}^{[\eta / \Delta h]} S\left(\cup_{i} X^{j \Delta h}\right) \Delta h} \\
& \cdot \sum_{j}^{\lfloor H / \Delta h\rfloor}\left(\sum_{m}^{\lfloor\xi / \Delta h\rfloor} S\left(X^{m \Delta h}\right) \Delta h\right) S\left(\bigcup_{i}\left(X_{i}^{j \Delta h} \oplus C_{i}\right) \Delta h\right) .
\end{aligned}
$$

Apparently, $V_{H}$ is a function of $H$, simply denoted as $V_{H}=F(H)$. It is easily to get the numerical solution $\widehat{H}$ of this equation by dichotomy. Here, $\hat{H}$ is the depth of inundation extent, which is also the depth of waterbody extent and water-invasion extent. The water-body extent $X^{\hat{H}}$ is approximately the corresponding crosssection $X^{\lfloor\hat{H} / \Delta h\rfloor \Delta h}$ within the set $X$. And water-invasion extent $Y^{\hat{H}}$ expanding from $X^{\hat{H}}$ is approximately described as follows:

$$
Y^{\lfloor\hat{H} / \Delta h\rfloor \Delta h}=\frac{\cup_{i}\left(X_{i}^{\lfloor\hat{H} / \Delta h\rfloor \Delta h} \oplus C_{i}^{\eta}\right) \sum_{m=0}^{\lfloor\hat{H} / \Delta h\rfloor} S\left(X^{m \Delta h}\right) \Delta h}{\sum_{j=0}^{\lfloor\eta \Delta h\rfloor} S\left(X^{j \Delta h}\right) \Delta h} .
$$

(iii) Usually, the estimation results of water-invasion extent are applied in analogue simulation or dynamic display. However, the lack of smoothness occasionally occurs at the boundaries because $Y^{\lfloor\hat{H} / \Delta h\rfloor \Delta h}$ is gained by point set, thus decreasing the effect of representation. The issue can be handled by opening operation of Mathematical Morphology. Specifically, we select the smallest structuring element $\operatorname{Min}\left[S\left(C_{i}^{\eta}\right)\right]$ of set $C_{i}^{\eta}$ as the opening operation conducting on $Y^{[\hat{H} / \Delta h] \Delta h}$, which eliminates the pointy part of water-invasion extent, removes narrow connections, and enhances the effect of visualization. The opening operation for smoothing water-invasion extent is as follows:

$$
\begin{aligned}
\hat{Y}^{\lfloor\hat{H} / \Delta h\rfloor \Delta h} & =Y^{\lfloor\hat{H} / \Delta h\rfloor \Delta h} \circ \operatorname{Min}\left[S\left(C_{i}^{\eta}\right)\right] \\
& =\left(Y^{\lfloor\hat{H} / \Delta h\rfloor \Delta h} \Theta \operatorname{Min}\left[S\left(C_{i}^{\eta}\right)\right]\right) \oplus \operatorname{Min}\left[S\left(C_{i}^{\eta}\right)\right] .
\end{aligned}
$$

\section{Experiments and Analyses}

In order to test the estimation method of inundation extent based on Mathematical Morphology, an experiment environment based on the Digital Earth platform was built with MODIS tile-pyramid RS images, GIS basin data, $0.6 \mathrm{~m}$ of QuickBird high-definition RS images, and $25 \mathrm{~m}$ of $26368 \times 26368$ DEM grid. DEM data and RS data were merged under the projection of EPSG4326, so the image units and projection of DEM and RS were unified in the same coordinate system. In the experiment environment, combining topographic map and thematic map, DEM data were horizontally subdivided in accordance with $\Delta h=1 \mathrm{~m}$. It created 986 DEM cross sections by the Kriging interpolation approach. Meanwhile, vegetation index, soil moisture index, and evaporation index obtained from RS data were aggregated to establish 412 equiangular subsets in the light of homogeneous properties.

Using the estimation method based on Mathematical Morphology and three other typical methods, we calculated the inundation extent caused by the water conservancy project, Hanjiang-to-Weihe River Water Transfer Project in China. This project built a reservoir, named Sanhekou Reservoir, with a total storage capacity of $6.81 \times 10^{9} \mathrm{~m}^{3}$ and the adjusted storage capacity of $5.5 \times 10^{9} \mathrm{~m}^{3}$. The main function of the reservoir is to regulate the inflow from the Ziwu River and transfer the inflow from the Han River through pumping stations. During the construction process of the Sanhekou Reservoir, the riverway closure led to water level increasing and lots of land submerged. The influence involves Shidunhe County, Shimudi County, Meizi County, Tongchewan Town, and Heba Town. More than 200 million yuan was considered to invest in the establishment of resettlement sites and the compensation for residents in the reservoir areas. Obviously, accurate inundation estimation will help to determine the amount of project investment, so we make the following estimates and analyses.

4.1. Accuracy Analysis. For the purpose of analyzing the accuracy of different methods, we estimated the inundation depth and inundation extent caused by the Sanhekou Reservoir at water volume of $1910 \times 10^{6} \mathrm{~m}^{3}$ and $2349 \times 10^{6} \mathrm{~m}^{3}$, as shown in Table 1 . HD represents the hydrodynamic method proposed in the literature [2], SF is the space-filling method put forward in the literature [20], LM is the method of simple expansion indicated in the literature [25], and MM represents the method proposed in this paper.

(i) When the water volume was $1910 \times 10^{6} \mathrm{~m}^{3}$ or $2349 \times 10^{6} \mathrm{~m}^{3}$, the area of inundation extent estimated by the HD, SF, and LM was all less than measurements, as shown in Table 1. After estimated the inundation extents under another 8 different water volumes, we found that the results obtained by the traditional methods of HD, SF, and LM were always lower than the actual result. The reason is those methods fail to take into account the waterinvasion process that plays a significant role in inundation estimation. As shown in Figure 2, the dark blue extent represents the water-body extent, and the adjacent blue-green extent denotes the water-invasion extent. Apparently, the traditional methods estimated inundation extent according to $V_{m}+V_{x}$ with the impact of water invasion ignored. In other words, the inundation extents obtained by the $\mathrm{HD}, \mathrm{SF}$, and $\mathrm{LM}$ were often larger than the water-body extent by the MM since the MM calculated water-body extent according to $V_{m}$. However, inundation extent actually includes waterinvasion extent and water-body extent. In geography, the mixture of water and soil causes more 
Table 1: Accuracy comparison of different methods.

\begin{tabular}{|c|c|c|c|c|c|c|c|}
\hline $\begin{array}{l}\text { Method (water } \\
\left.\text { volume }\left(10^{6} \mathrm{~m}^{3}\right)\right)\end{array}$ & $\begin{array}{c}\text { Inundation } \\
\text { extent } \\
\left(\mathrm{km}^{2}\right)\end{array}$ & $\begin{array}{l}\text { Inundation } \\
\text { depth }(\mathrm{m})\end{array}$ & $\begin{array}{c}\text { Absolute error of } \\
\text { inundation extent } \\
\left(\mathrm{km}^{2}\right)\end{array}$ & $\begin{array}{c}\text { Relative error of } \\
\text { inundation } \\
\text { extent (\%) }\end{array}$ & $\begin{array}{l}\text { Absolute error of } \\
\text { inundation depth } \\
(\mathrm{m})\end{array}$ & $\begin{array}{c}\text { Relative error of } \\
\text { inundation depth } \\
(\%)\end{array}$ & $\begin{array}{c}\text { Time } \\
\text { consumed } \\
(\mathrm{ms})\end{array}$ \\
\hline HD (1910) & 6.310 & 601.672 & 0.071 & 1.113 & 2.665 & 0.441 & 45912 \\
\hline SF (1910) & 6.219 & 600.743 & 0.162 & 2.539 & 3.594 & 0.595 & 481 \\
\hline LM (1910) & 6.353 & 601.856 & 0.028 & 0.439 & 2.481 & 0.411 & 566 \\
\hline MM (1910) & 6.395 & 606.128 & 0.014 & 0.219 & 1.791 & 0.296 & 832 \\
\hline HD (2349) & 7.643 & 618.977 & 0.139 & 1.786 & 4.952 & 0.794 & 51150 \\
\hline SF (2349) & 7.560 & 617.263 & 0.222 & 2.853 & 6.666 & 1.068 & 526 \\
\hline LM (2349) & 7.693 & 619.849 & 0.089 & 1.144 & 4.080 & 0.654 & 601 \\
\hline MM (2349) & 7.831 & 625.201 & 0.049 & 0.630 & 1.272 & 0.204 & 908 \\
\hline
\end{tabular}

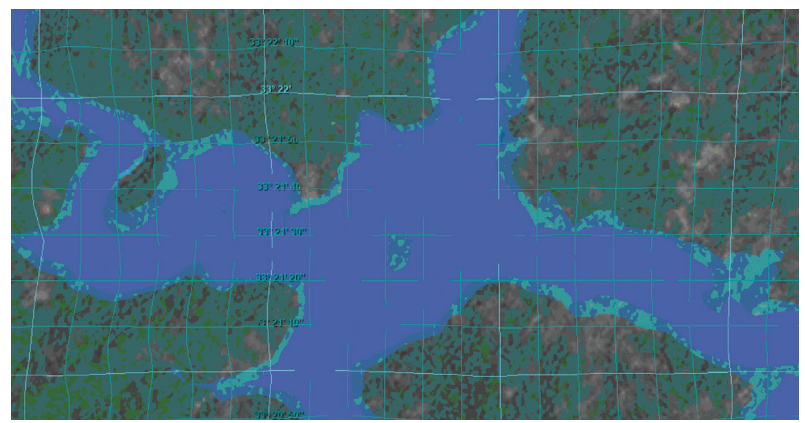

Figure. 2: Experiment environment based on the Digital Earth platform.

inundation than the water itself, so the water $V_{x}$ invading the soil surrounding water-body extent enlarges the inundation extent. That is to say, corresponding to the water $V_{x}$, the inundation extent estimated by the traditional methods is smaller than that estimated by the MM. Thus, the inundation extents estimated by the HD, SF, and LM were smaller than that (including water-invasion extent and water-body extent) estimated by the MM.

(ii) The MM method proposed in this paper showed relatively higher estimation accuracy than the other methods. Its absolute error of inundation estimation of the MM method was less than $0.05 \mathrm{~km}^{2}$, and the relative error of that was less than $0.8 \%$. The errors of the MM method were only half that of the other methods. Unlike the methods of HD, SF, and LM, the inundation extent and inundation depth obtained by the MM method were a little bit larger than actual measurements. This suggests that the introduction of Mathematical Morphology improves the accuracy of inundation estimation. The MM method approximated to the result of the actual measurement is superior to the others and provides more safe projections to avoid underestimating the damage of inundation.

(iii) After the smoothing of opening operation was adopted in the water-invasion extent, the area of inundation extent under those two different water volumes became $6.398 \mathrm{~km}^{2}$ and $7.835 \mathrm{~km}^{2}$, manifesting a further increase in error. Although more error reduced the estimation accuracy, it was less than $0.005 \mathrm{~km}^{2}$ which only accounts for $0.08 \%$ of the area of inundation extent and can be ignored. However, the smoothing of opening operation makes the inundation extents match well with the boundaries and RS data. It improves the effect of visualization when simulating the inundation process or displaying the inundation extent.

(iv) The HD method took $45 \mathrm{~s}$ for calculation, which made it impossible to carry out real-time simulation. In contrast, although the MM method spent twice as much time as the SF method did, it was able to control the time consumption within the limit of $1 \mathrm{~s}$. It was clear that the MM method possessed high timeliness for multiple-frame inundation simulation and great efficiency for dynamic simulation.

4.2. Influential Factor Analysis. The MM method, implemented by horizontal subdivision and equiangular subsets, estimates inundation extent under given water volume with DEM data. Therefore, the estimation accuracy largely depends on the factors including water volume, the number of horizontal subdivisions, and the number of equiangular subsets.

4.2.1. Water Volume. As for the HD, SF, and LM methods, the larger the water volume is, the more influences the underlying surface and landforms would impose, thereby leading to more errors. As shown in Figure 3, the absolute errors increased with the increase in water volume. The relative error surpassed $2 \%$ at the water volume of $2880 \times 10^{6} \mathrm{~m}^{3}$ which barely met the accuracy need of disaster evaluation. Particularly, the exceeding amount of water complicated the HD method and dramatically made the analogue simulation less effective. By contrast, the MM method is based on the expansion of water body, so the subdivision of the expansion process will be more compact as the water volume increases. Thus, equiangular subsets with more homogeneous properties were generated, which partially reduced the impact of environmental factors and helped to acquire more accurate results. As shown in Figure 4, the effect of water volume on accuracy was not significant when the MM method was adopted. 


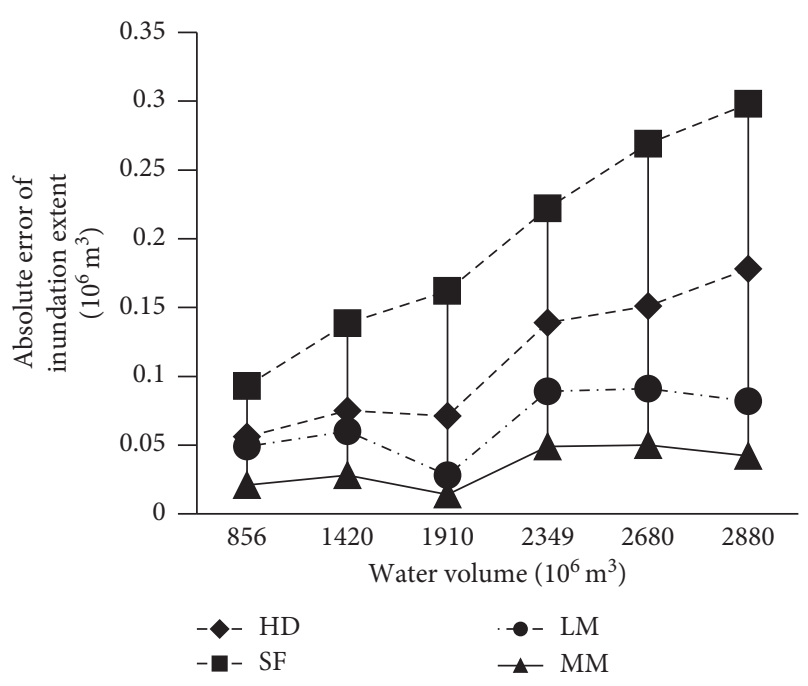

Figure 3: Absolute errors under different water volumes.

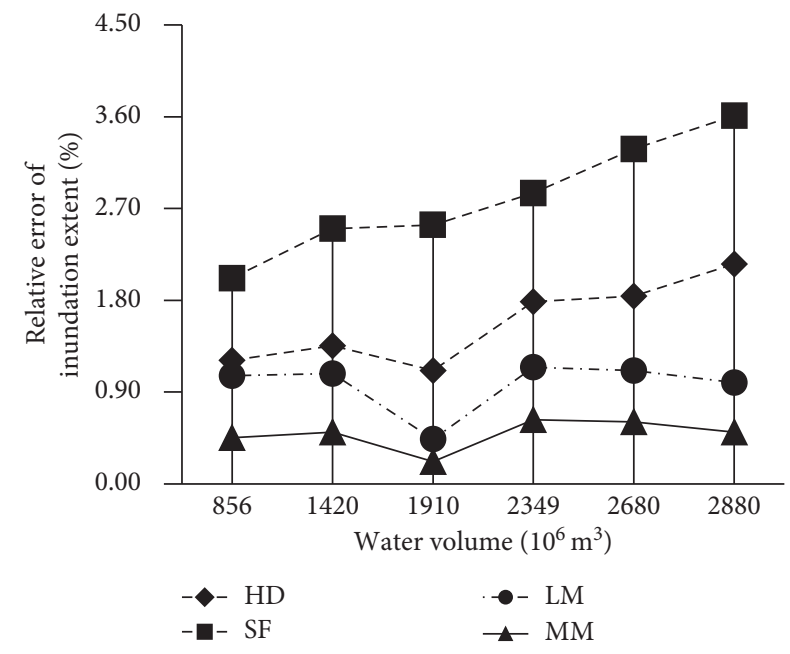

Figure 4: Relative errors under different water volumes.

4.2.2. Number of Horizontal Subdivisions. Its influence on the MM method appeared as a concave curve, shown in Figure 5. Excessively large number of horizontal subdivisions enlarged the differences between structuring elements, while small subdivisions led to inadequate use of the data resources of landforms. Both of them resulted to lower estimation accuracy. Therefore, in order to ensure sufficient timeliness and simulation accuracy, DEM data have to reach high enough grid accuracy. The optimal number of subdivisions is the minimum extent difference of two neighboring cross sections divided by $\Delta h$, i.e., $\min _{i \neq j}\left(\mid X^{i \Delta h}-\right.$ $\left.X^{j \Delta h} \mid\right) / \Delta h$, so as to make sure that the minimum difference can be recognized.

4.2.3. Number of Equiangular Subsets. The equiangular subsets are derived from aggregating influential factors of landforms. According to the MM method, too small number of equiangular subsets results in fewer structuring elements and fewer templates available for the expansion operation.

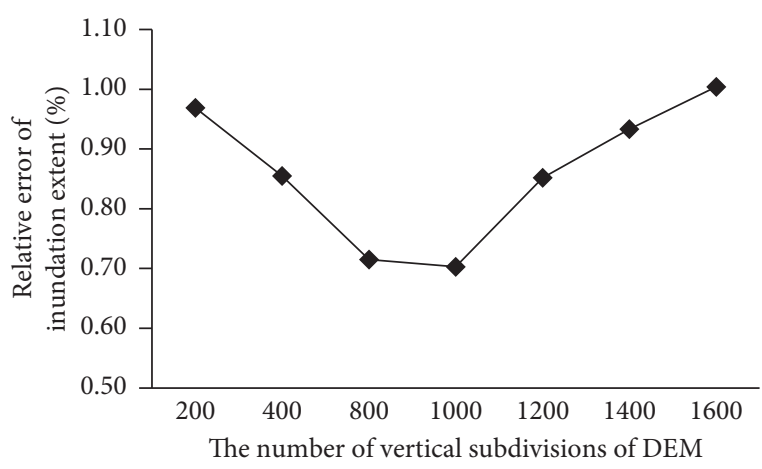

FIGURE 5: Relative errors under different horizontal subdivisions.

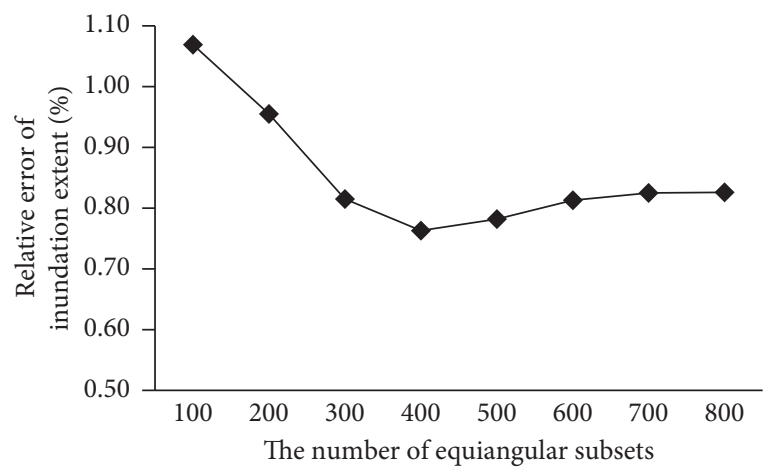

FIgURE 6: Relative errors under different equiangular subsets.

Consequently, it gives rise to insufficient expansion operation with homogeneous properties, reduces the expansion abilities from water-body extent to water-invasion extent, and then diminishes estimation accuracy. On the contrary, excessive number of equiangular subsets increases the computational load and the number of structuring elements, thus leading to redundant expansion operation of the same region. Therefore, the estimation result was much larger than the actual measurement, as shown in Figure 6.

\section{Conclusion}

Inundation estimation is an important work of disaster analysis, which determines the scope and magnitude of the disaster. Focusing on the problem of insufficient accuracy of inundation estimation, we proposed an estimation method based on Mathematical Morphology. The method takes inundation extent as the sum of water-body extent and water-invasion extent. With DEM data, water-body extent was calculated by space-filling method, but it adopted horizontal subdivisions rather than vertical subdivisions. Water-invasion extent was expanded from water-body extent by structuring elements and calculated with equiangular subsets. The experimental results show that the MM method proposed in this paper has higher accuracy than the typical hydrodynamic method and space-filling method. The estimation accuracy of the MM method is largely relative to the factors including water volume, the number of horizontal subdivisions, and the number of equiangular subsets. Although the MM method gives estimations of inundation 
extent and inundation depth with a little bit larger than actual measurements, it avoids underestimating the damage of inundation. It is worth mentioning that the expansion operation of water-invasion extent relies on the equiangular subsets and structuring elements created by RS data which reflecting landform features, so the improvement of accuracy of RS image data can further improve the estimation result.

\section{Data Availability}

The program code and data that support the plots discussed within this paper are available from the corresponding author upon request.

\section{Conflicts of Interest}

The authors declare that they have no conflicts of interest.

\section{Acknowledgments}

Jianxun Li gratefully acknowledges the support of the Basic Research Project of Natural Science (Research and Implementation of Assistant Decision-making for Immigrant of Water Conservancy Projects under the 3S Technology, no. 2014JM9365), Shaanxi Province, China.

\section{References}

[1] M. S. Horritt, D. C. Mason, and A. J. Luckman, "Flood boundary delineation from synthetic aperture radar imagery using a statistical active contour model," International Journal of Remote Sensing, vol. 22, no. 13, pp. 2489-2507, 2001.

[2] N. Y. Nguyen, Y. Ichikawa, and H. Ishidaira, "Estimation of inundation depth using flood extent information and hydrodynamic simulations," Hydrological Research Letters, vol. 10, no. 1, pp. 39-44, 2016.

[3] P. Bates, C. Sampson, A. Smith et al., "Validation of a global hydrodynamic flood inundation model against high resolution observation data of urban flooding," in Proceedings of the EGU General Assembly Conference Abstracts, vol. 17, pp. 2-8, Vienna, Austria, April 2015.

[4] M. Podhoranyi, S. Kuchar, and A. Portero, "Flood evolution assessment and monitoring using hydrological modelling techniques: analysis of the inundation areas at a regional scale," IOP Conference Series: earth and Environmental Science, vol. 39, no. 1, Article ID 012043, 2016.

[5] F. Ilorme, Development of a Physically-Based Method for Delineation of Hydrologically Homogeneous Regions and Flood Quantile Estimation in Ungauged Basins via the Index Flood, Michigan Technological University, Houghton, Michigan, 2011.

[6] M. L. Follum, A. A. Tavakoly, J. D. Niemann, and A. D. Snow, "AutoRAPID: a model for prompt streamflow estimation and flood inundation mapping over regional to continental extents," JAWRA Journal of the American Water Resources Association, vol. 53, no. 2, pp. 280-299, 2017.

[7] A. T. Murphy, B. Gouldby, S. J. Cole, R. J. Moore, and H. Kendall, "Real-time flood inundation forecasting and mapping for key railway infrastructure: a UK case study," E3S Web of Conferences, vol. 7, 2016.

[8] S. Gobeyn, A. Van Wesemael, J. Neal et al., "Impact of the timing of a SAR image acquisition on the calibration of a flood inundation model," Advances in Water Resources, vol. 100, pp. 126-138, 2017.

[9] D. P. Patel, J. A. Ramirez, P. K. Srivastava, M. Bray, and D. Han, "Assessment of flood inundation mapping of Surat city by coupled $1 \mathrm{D} / 2 \mathrm{D}$ hydrodynamic modeling: a case application of the new HEC-RAS 5," Natural Hazards, vol. 89, no. 1, pp. 93-130, 2017.

[10] M. Faghih, M. Mirzaei, J. Adamowski, J. Lee, and A. El-Shafie, "Uncertainty estimation in flood inundation mapping: an application of non-parametric bootstrapping," River Research and Applications, vol. 33, no. 4, pp. 611-619, 2017.

[11] S. Afshari, A. A. Tavakoly, M. A. Rajib et al., "Comparison of new generation low-complexity flood inundation mapping tools with a hydrodynamic model," Journal of Hydrology, vol. 556, pp. 539-556, 2018.

[12] S. Cohen, G. R. Brakenridge, A. Kettner et al., "Estimating floodwater depths from flood inundation maps and topography," JAWRA Journal of the American Water Resources Association, vol. 54, no. 4, pp. 847-858, 2018.

[13] N. S. Noman, E. J. Nelson, and A. K. Zundel, "Review of automated floodplain delineation from digital terrain models," Journal of Water Resources Planning and Management, vol. 127, no. 6, pp. 394-402, 2001.

[14] A. M. Dewan, T. Kumamoto, and M. Nishigaki, "Flood hazard delineation in greater Dhaka, Bangladesh using an integrated GIS and remote sensing approach," Geocarto International, vol. 21, no. 2, pp. 33-38, 2006.

[15] D. C. Mason, P. D. Bates, and J. T. Dall'Amico, "Calibration of uncertain flood inundation models using remotely sensed water levels," Journal of Hydrology, vol. 368, no. 1-4, pp. 224-236, 2009.

[16] S. Kamontum, Fusion of Landsat-7, IRS-1D and Radarsat-1 Data for Flood Delineation, University of Florida, Gainesville, Fl, USA, 2009.

[17] F. Dottori and E. Todini, "Developments of a flood inundation model based on the cellular automata approach: testing different methods to improve model performance," Physics and Chemistry of the Earth, Parts $A / B / C$, vol. 36, no. 7-8, pp. 266-280, 2011.

[18] R. Charrier and Y. Li, "Assessing resolution and source effects of digital elevation models on automated floodplain delineation: a case study from the Camp Creek Watershed, Missouri," Applied Geography, vol. 34, pp. 38-46, 2012.

[19] S. Saksena and V. Merwade, "Incorporating the effect of DEM resolution and accuracy for improved flood inundation mapping," Journal of Hydrology, vol. 530, pp. 1-52, 2015.

[20] J. Teng, J. Vaze, D. Dutta, and S. Marvanek, "Rapid inundation modelling in large floodplains using LiDAR DEM," Water Resources Management, vol. 29, no. 8, pp. 2619-2636, 2015.

[21] J. Sopelana, L. Cea, and S. Ruano, "A continuous simulation approach for the estimation of extreme flood inundation in coastal river reaches affected by meso- and macrotides," Natural Hazards, vol. 93, no. 3, pp. 1337-1358, 2018.

[22] J. Teng, A. J. Jakeman, J. Vaze, B. F. W. Croke, D. Dutta, and S. Kim, "Flood inundation modelling: a review of methods, recent advances and uncertainty analysis," Environmental Modelling \& Software, vol. 90, pp. 201-216, 2017.

[23] J. F. Rosser, D. G. Leibovici, and M. J. Jackson, "Rapid flood inundation mapping using social media, remote sensing and topographic data," Natural Hazards, vol. 87, no. 1, pp. 103120, 2017.

[24] M. O. Sghaier, S. Foucher, and R. Lepage, "River extraction from high-resolution SAR images combining a structural feature set and mathematical morphology," IEEE Journal of 
Selected Topics in Applied Earth Observations and Remote Sensing, vol. 10, no. 3, pp. 1025-1038, 2016.

[25] H. Vernieuwe, B. De Baets, and N. E. C. Verhoest, "A mathematical morphology approach for a qualitative exploration of drought events in space and time," International Journal of Climatology, no. 6, pp. 1-14, 2019.

[26] F. W. Li, X. N. Zhang, and C. W. Du, "Study on flood submergence based on GIS and mathematical morphology," Advances in Science and Technology of Water Resources, vol. 25, no. 6, pp. 14-24, 2005. 


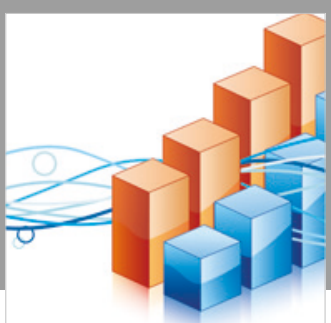

Advances in

Operations Research

\section{-n-m}
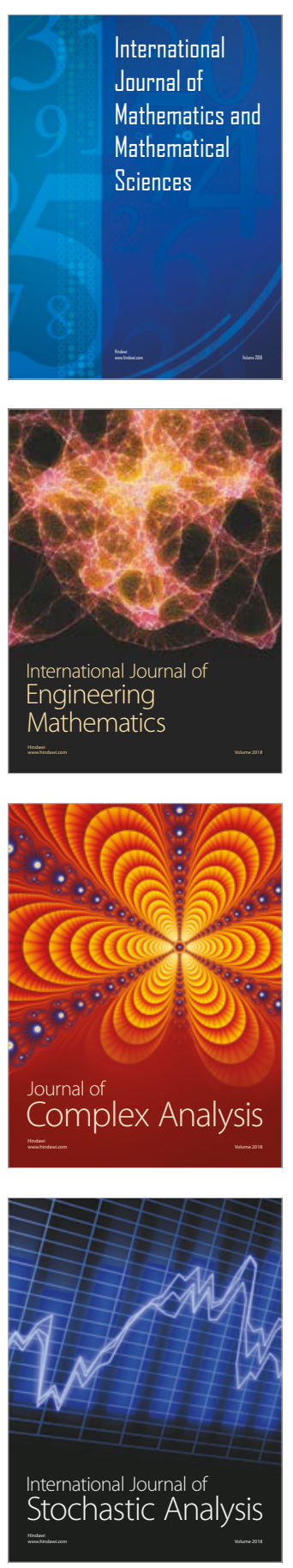
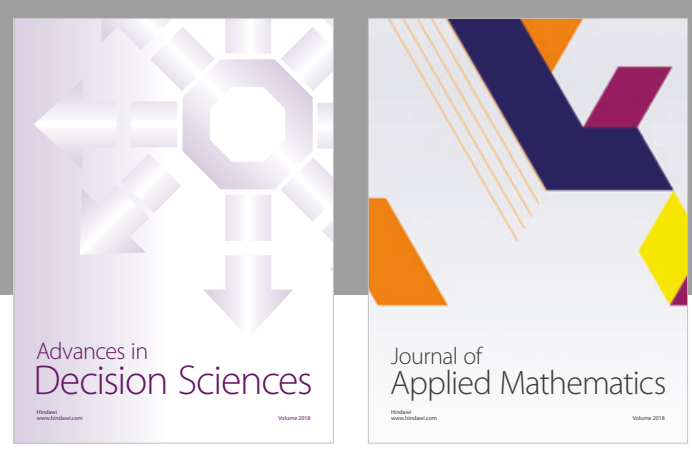

Journal of

Applied Mathematics
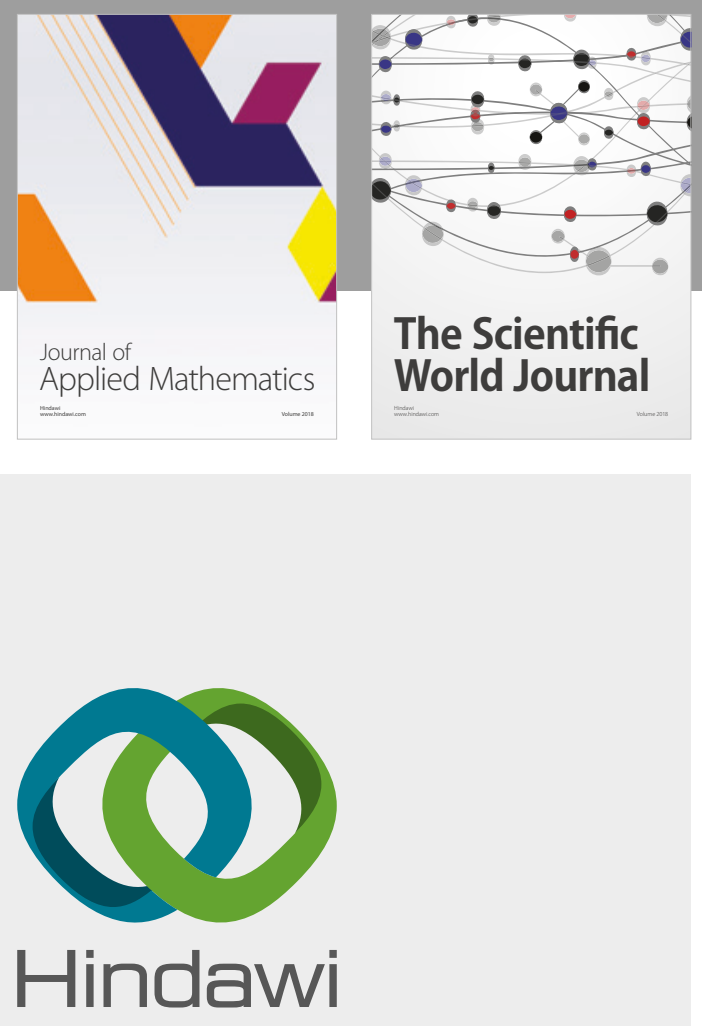

Submit your manuscripts at

www.hindawi.com

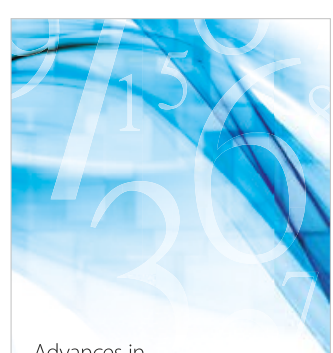

Advances in
Numerical Analysis
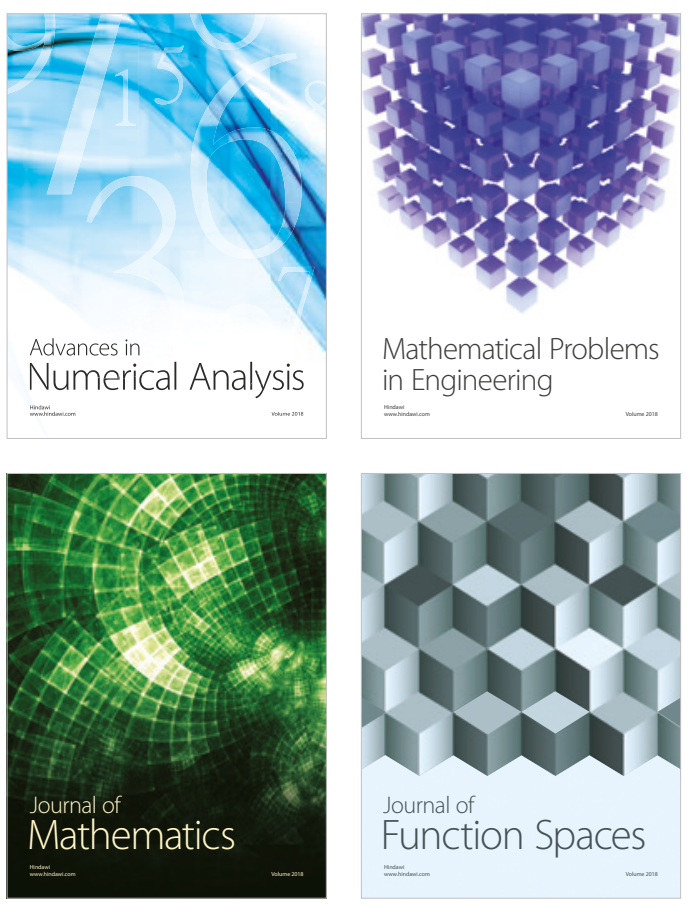

Mathematical Problems in Engineering

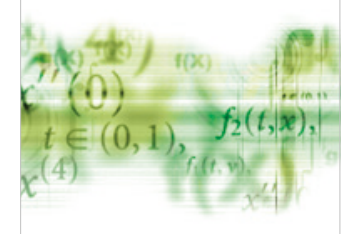

International Journal of

Differential Equations

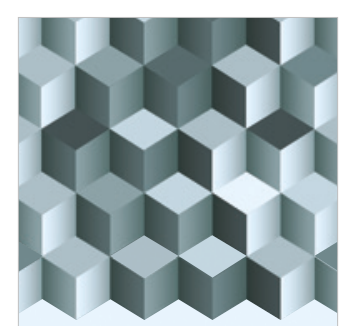

Journal of

Function Spaces

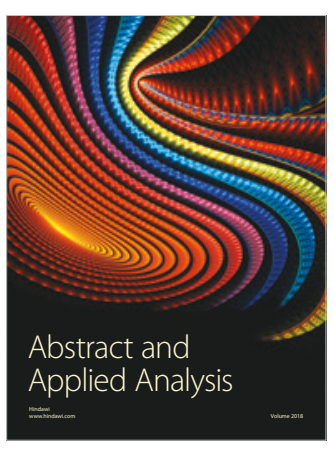

The Scientific

World Journal

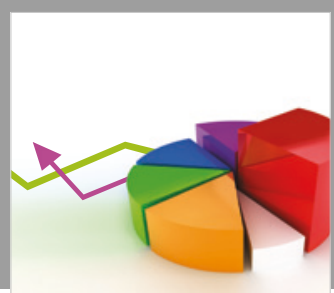

Journal of

Probability and Statistics
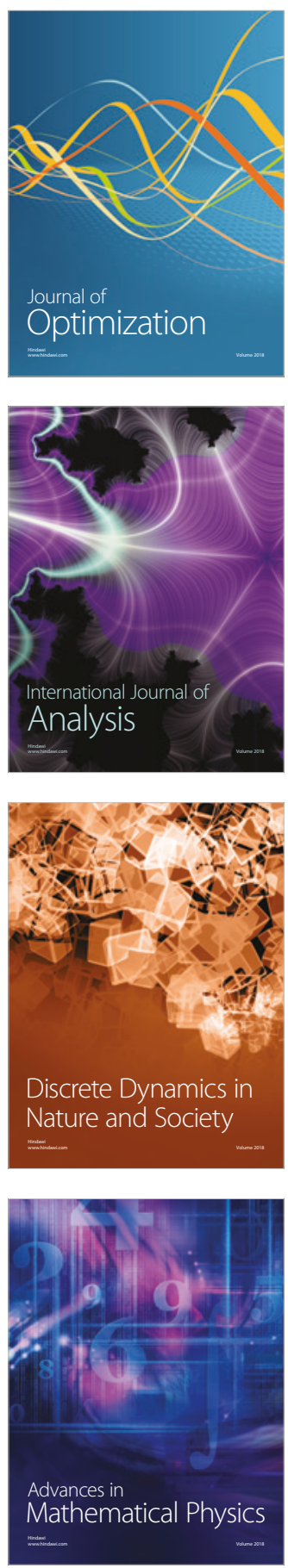